OPEN ACCESS

Edited by:

Tae-Hee Cho,

Hospices Civils de

Lyon, France

Reviewed by: Simone Beretta,

Azienda Ospedaliera

San Gerardo, Italy

Richard Leigh,

National Institutes of Health,

United States

*Correspondence:

Hannah Fuhrer

hannah.fuhrer@uniklinik-freiburg.de

Specialty section:

This article was submitted to Stroke,

a section of the journal

Frontiers in Neurology

Received: 19 May 2017

Accepted: 26 July 2017

Published: 10 August 2017

Citation:

Fuhrer H, Günther A, Zinke J and

Niesen W-D (2017) Optimizing Cardiac Out-Put to Increase Cerebral Penumbral Perfusion in Large Middle Cerebral Artery Ischemic Lesion-OPTIMAL Study.

Front. Neurol. 8:402.

doi: 10.3389/fneur.2017.00402

\section{Optimizing Cardiac Out-Put to Increase Cerebral Penumbral Perfusion in Large Middle Cerebral Artery Ischemic Lesion - OPTIMAL Study}

\author{
Hannah Fuhrer ${ }^{1 *}$, Albrecht Günther ${ }^{2}$, Jan Zinke ${ }^{2}$ and Wolf-Dirk Niesen ${ }^{1}$ for the IGNITE \\ Study Group
}

${ }^{1}$ Department of Neurology, Albert Ludwig University of Freiburg, Freiburg, Germany, ${ }^{2}$ Department of Neurology, Friedrich Schiller University Jena, Jena, Germany

Introduction: In unsuccessful vessel recanalization, clinical outcome of acute stroke patients depends on early improvement of penumbral perfusion. So far, mean arterial blood pressure (MAP) is the target hemodynamic parameter. However, the correlations of MAP to cardiac output (CO) and cerebral perfusion are volume state dependent. In severe subarachnoid hemorrhage, optimizing $\mathrm{CO}$ leads to a reduction of delayed ischemic neurological deficits and improvement of clinical outcome. This study aims to investigate the effect of standard versus advanced cardiac monitoring with optimization of $\mathrm{CO}$ on the clinical outcome in patients with large ischemic stroke.

Methods and analysis: The OPTIMAL study is a prospective, multicenter, open, into two arms (1:1) randomized, controlled trial. Sample size estimate: sample sizes of 150 for each treatment group (300 in total) ensure an $80 \%$ power to detect a difference of $16 \%$ of a dichotomized level of functional clinical outcome at 3 months at a significance level of 0.05. Study outcomes: the primary endpoint is the functional outcome at 3 months. The secondary endpoints include functional outcome at 6 months follow-up, and complications related to hemodynamic monitoring and therapies.

Discussion: The results of this trial will provide data on the safety and efficacy of advanced hemodynamic monitoring on clinical outcome.

Ethics and dissemination: The trial was approved by the leading ethics committee of Freiburg University, Germany $(438 / 14,2015)$ and the local ethics committees of the participating centers. The study is performed in accordance with the Declaration of Helsinki and the guidelines of Good Clinical Practice. It is registered in the German Clinical Trial register (DRKS; DRKS00007805). Dissemination will include submission to peer-reviewed professional journals and presentation at congresses. Hemodynamic monitoring may be altered in a specific stroke patient cohort if the study shows that advanced monitoring is safe and improves the functional outcome.

Keywords: acute stroke therapy, hemodynamics, cardiac output, cerebral perfusion, functional outcome, cerebral infarction 


\section{INTRODUCTION}

Acute therapy in stroke focuses on restoring and optimizing the cerebral perfusion $(\mathrm{CP})$ to reduce the final infarction volume and improve the clinical outcome $(1,2)$. Guidelines advocate early recanalization measures by intravenous thrombolysis and/or thrombectomy as a standard of care. In insufficient and unsuccessful vessel recanalization, clinical outcome of acute stroke patients depends on early improvement of penumbral perfusion. The individual evolution of good collateral vessels is a crucial factor for the development and maintainability of penumbral perfusion (1). So far, mean arterial blood pressure (MAP) is the standard target parameter for improving CP being a readily accessible parameter on one hand and expected to be related to CP due to vascular autoregulation on the other (3-5). It has been shown that an increase of MAP levels can reduced the infarction volume and better functional outcome due to improved penumbral perfusion (6). Physically, MAP itself results from a product of cardiac output (CO) and systemic vascular resistance (SVR; 6).

However, former studies have shown that correlation of MAP, and organ perfusion depends on volume state $(7,8)$, whereas CO has a linear correlation with perfusion $(5,9)$. Especially in the critically ill, optimized CO correlated with increased organ oxygen delivery, and thus leads to improvement of clinical outcome (10).

In patients with severe subarachnoid hemorrhage (SAH) increased CO during constant, MAP levels lead to a higher cerebral blood flow reducing the rate of delayed cerebral ischemia $(11,12)$ and improving the functional outcome (13).

The Optimizing cardiac out-Put To Increase cerebral penumbral perfusion in large Middle cerebral Artery ischemic Lesion (OPTIMAL) study aims to investigate the effect of advanced cardiac monitoring with optimization of $\mathrm{CO}$ levels in stroke patients. We hypothesize that advanced monitoring reduces the infarction volume and improves clinical outcome.

\section{METHODS AND ANALYSIS}

\section{Design}

The OPTIMAL study is a prospective, multicenter, open, twoarmed, randomized, and outcome-blinded trial. The participating centers are part of the IGNITE network.

\section{Patient Population - Inclusion and Exclusion Criteria}

All subjects with an ischemic stroke are eligible for participation if the following inclusion criteria are met:

- Ischemic stroke $\geq 30 \%$ of the territory of the middle cerebral artery (visual assessment of CT or MR scans).

- Age between 18 and 85 years.

- Within $12 \mathrm{~h}$ of symptom onset/12 h from time last seen well.

- Patients without recanalization measures: inclusion within $3 \mathrm{~h}$ after hospital admission.
- Patients with recanalization therapy (i.v. thrombolysis, thrombectomy, or both): inclusion within $3 \mathrm{~h}$ after cessation of recanalization measures and persistent neurological deficit [with National Institute of Health Stroke Scale (NIH-SS) $>10$ points]. If patients are in a sedated state and cannot be clinically monitored, the vessel occlusion must be persistent (depicted by angiography or sonography).

- Written informed consent by the subject herself/himself, her/ his legal representative or written/oral consent by a close relative or friend according to the patient's presumed will and interest of participating in this study.

Subjects will be excluded from the trial if any of the following criteria is fulfilled:

- Patients receiving recanalization measures: patients presenting with NIH-SS $\leq 10$ points during recanalization measures or within $3 \mathrm{~h}$ after cessation of recanalization measures.

- Intracerebral hemorrhage.

- Premorbid modified Rankin (mRS) score $\geq 2$.

- Severe comorbidities or malignant disease leading to a relevant impact on the 3 months outcome.

\section{Randomization}

Subjects are randomly assigned to standard monitoring or standard plus advanced monitoring using an online-randomization tool (www.randomizer.at). Randomization allocates patients 1:1 to one of the treatment arms; randomization factors are age, gender, hemisphere, NIH-SS, and recanalization measurements.

\section{Treatment or Intervention}

All participating centers have adequate experience in the intensive care management of acute ischemic stroke.

\section{Control Arm: Standard Monitoring and Therapy}

All patients receive best medical care according to the standards based on the recommendations of the German stroke association. MAP levels of $\geq 80 \mathrm{mmHg}$ have to be achieved for $48 \mathrm{~h}$; volume (crystalloids) or catecholamines (noradrenaline and dobutamine) are to be administered if needed. According to our hospital standard of care, systolic blood pressure levels are limited to $160 \mathrm{mmHg}$ in patients who underwent recanalization measures.

\section{Treatment Arm: Standard Plus Advanced Monitoring and Therapy}

Patients receive standard plus advanced cardiac monitoring. Those monitoring systems ("Vigileo," "PiCCO") perform an analysis of the arterial pulse contour and calculate a cardiac index (CI; index results from $\mathrm{CO}$ and stroke volume related to body surface). Optimizing both parameters with MAP levels $\geq 80 \mathrm{mmHg}$ and CI ranging between $\geq 3.0$ and $\leq 4.5$ has to be achieved for $48 \mathrm{~h}$ with volume (crystalloids) or catecholamines (noradrenaline and dobutamine) administration if necessary. According to our hospital standard of care, systolic blood pressure levels are limited to $160 \mathrm{mmHg}$ in patients who underwent recanalization measures. 
The monitoring systems calculate further parameters, which can be used to guide hemodynamic therapy: as a preload indicator the stroke volume variety (SVV) is calculated by (SVmax - SVmin)/SVmean with SVV $>10 \%$ being a sign of volume depletion. The SVR index indicates vessel dilation and/or volume depletion [normal SVRI levels 1,700-2,400 dyn s/cm 5 $\left.\mathrm{m}^{2}(13)\right]$. So, in patients with CI $<3.0$, crystalloids should be administered as a first step of hemodynamic treatment until SVV $<10 \%$. If this measure does not increase the $\mathrm{CI}>3$, noradrenaline, a peripheral vasoconstrictor, according to SVR levels should be used. Dobutamine, as a positive inotropic substance, can be used as last option.

\section{Primary Outcomes}

The primary outcome is a dichotomized mRS score after 3 months score $0-3$ versus $4-6$ is compared within the treatment groups.

\section{Secondary Outcomes}

Dichotomized outcome of mRS score 0-3 versus 4-6 after 6 months, as well as $0-2$ versus 3-6 after 3 and 6 months, change of NIH-SS on day $1,2,3$, and $10 \pm 3$ are analyzed. Final infarction volume is calculated. Monitoring parameters (CI and MAP levels) as well as amount of fluid administration and catecholamines are analyzed. Furthermore, safety assessments include the following: in hospital mortality, days of hospital stay, number of secondary intracerebral hemorrhages, and duration and quantity of catecholamine treatment.

\section{Data Monitoring Body}

Data will be recorded in every participating center, and all data will be finally analyzed in the principle center (University Freiburg). Safety parameters will be thoroughly assessed (c.f. secondary outcomes).

\section{Sample Size Estimates}

Sample size calculations are based on the study of Mutoh et al. (13). Sample sizes of 150 for each treatment group (300 in total) ensure an $80 \%$ power to detect a difference of $16 \%$ in proportions of patients with mRS scores of $0-3$ at 3 months at a significance level of 0.05 .

\section{Statistical Analyses}

Statistical analysis will be performed as intention to treat. Summary statistics will be used for descriptive analysis. $P$-values for group comparisons and corresponding 95\% confidence intervals will be calculated using two-sample $t$-testing, the Mann-Whitney $U$-test, or $\chi^{2}$-testing as applicable.

\section{Study Organization and Funding}

The steering committee consists of the project leader Wolf-Dirk Niesen and the principle investigators of the participating centers. The study received funding for the patient randomization by the German Society for Neurointensive Care and Emergency Medicine (DGNI). Besides this, the study is exclusively driven by internal means of the participating centers.

\section{Stepwise Procedures}

Patients with acute ischemic stroke are screened for in- and exclusion criteria when admitted to the hospital. Randomization takes place within $3 \mathrm{~h}$ when no acute recanalization measures take place or within $3 \mathrm{~h}$ after cessation of recanalization measures. From then on, monitoring and optimizing of hemodynamic parameters are applied for the next $48 \mathrm{~h}$ in both groups. Data of NIH-SS scores are collected on day $1,2,3$, and $10 \pm 3$, the data of mRS scores at time of discharge and after 3 and 6 months. A follow-up MRI is performed on day $10 \pm 3$.

\section{Anticipated Results}

The OPTIMAL study aims to investigate the effect of advanced hemodynamic monitoring with optimization of $\mathrm{CO}$ levels in stroke patients. We anticipate an advanced monitoring to improve clinical long-term outcome and reduce the infarction volume.

\section{DISCUSSION}

The penumbra concept describes tissue at risk due to hypoxemia around the infarction core. The fate of the penumbral tissue seems to be individually variable and depends on slight differences in perfusion (14). Cerebral collaterals are dynamically recruited after arterial occlusion. Patients being able to develop good collateral flow and therefore restore the penumbra longer seem to develop smaller infarctions. Furthermore, they seem to benefit most from perfusion strategies (1). Based on this penumbra concept, the timeframe of a maximum of $12 \mathrm{~h}$ after symptom onset until inclusion in this study was adopted (2).

Patients being treated with an i.v. thrombolysis and/or thrombectomy are excluded from this study within the first $3 \mathrm{~h}$ after completion of recanalization therapy due to the increased risk of secondary hemorrhages (15). The bloodbrain barrier is abnormally permeable, and treatment measures in the very early acute phase may precipitate or enhance hemorrhagic transformation (16). Therefore, patients can be included into this trial $3 \mathrm{~h}$ after the cessation of recanalization measures.

With focus on outcome parameters and since there are no hemodynamic data on severely ill stroke patients, we based our study on data of Ref. (13). They were able to show that advanced hemodynamic monitoring can reduce the incidence of DCI and therefore patients with severe SAH benefited from advanced monitoring with a significant better outcome with a difference concerning independency (mRS 0-3) of 16\% (13). Based on results of former studies that standard monitoring poorly reflects actual circulating blood volume and organ perfusion but fluid status effects brain perfusion $(5,7,8,13)$, we hypothesize that advanced hemodynamic monitoring can reduce the final infarction volume in large ischemic stroke. Thus, we adopted the dichotomized primary endpoint of $\mathrm{mRS}$ score at 3 months aiming to show a positive effect of advanced hemodynamic monitoring with a shift to a more favorable outcome. 
We believe that results of OPTIMAL will provide reliable data on hemodynamic monitoring and functional outcome in large ischemic stroke, thereby forming the basis of subsequent future studies.

\section{ETHICS STATEMENT}

The trial was approved by the leading ethics committee of Freiburg University, Germany (Reference Number: 438/14, date of approval: 29.01.2015) and the local ethics committees of the participating centers. The study is performed in accordance with the Declaration of Helsinki and its subsequent amendments and the guidelines of Good Clinical Practice. The study is registered in the German Clinical Trial register (DRKS; DRKS00007805). Dissemination will include submission of study results to peerreviewed professional journals and presentation at congresses. Hemodynamic monitoring may be altered in stroke patients

\section{REFERENCES}

1. Manning NW, Campbell BC, Oxley TJ, Chapot R. Acute ischemic stroke: time, penumbra, and reperfusion. Stroke (2014) 45(2):640-4. doi:10.1161/ STROKEAHA.113.003798

2. Davis S, Donnan GA. Time is penumbra: imaging, selection. Cerebrovasc Dis (2014) 38(1):59-72. doi:10.1159/000365503

3. Krainik A, Villien M, Troprès I, Attyé A, Lamalle L, Bouvier J, et al. Functional imaging of cerebral perfusion. Diagn Interv Imaging (2013) 94(12):1259-78. doi:10.1016/j.diii.2013.08.004

4. Reinhard M, Rutsch S, Lambeck J, Wihler C, Czosnyka M, Weiller C, et al. Dynamic cerebral autoregulation associates with infarct size and outcome after ischemic stroke. Acta Neurol Scand (2012) 125(3):156-62. doi:10.1111/j.1600-0404.2011.01515.x

5. Fuhrer H, Weiller C, Niesen W-D. Is mean arterial pressure the best parameter in ischemic stroke? Clin Case Rep (2016) 4(3):236-9. doi:10.1002/ccr3.491

6. Hillis AE, Ulatowski JA, Barker PB, Torbey M, Ziai W, Beauchamp NJ, et al. A pilot randomized trial of induced blood pressure elevation: effects on function and focal perfusion in acute and subacute stroke. Cerebrovasc Dis (2003) 16:236-46. doi:10.1159/000071122

7. Marik PE, Monnet X, Teboul J-L. Hemodynamic parameters to guide fluid therapy. Ann Intensive Care (2011) 1(1):1. doi:10.1186/2110-5820-1-1

8. Linton DM, Gilon D. Advances in noninvasive cardiac output monitoring. Ann Card Anaesth (2002) 5(2):141-8.

9. Ogoh S, Brothers RM, Barnes Q, Eubank WL, Hawkins MN, Purkayastha S, et al. The effect of changes in cardiac output on middle cerebral artery mean blood velocity at rest and during exercise. J Physiol (2005) 569(Pt 2):697-704. doi:10.1113/jphysiol.2005.095836

10. Kern JW, Shoemaker WC. Meta-analysis of hemodynamic optimization in high-risk patients. Crit Care Med (2002) 30(8):1686-92. doi:10.1097/ 00003246-200208000-00002

11. Yoneda H, Nakamura T, Shirao S, Tanaka N, Ishihara H, Suehiro E, et al. Multicenter prospective cohort study on volume management after subarachnoid hemorrhage: hemodynamic changes according to severity of or in a specific stroke patient cohort if the study shows that advanced monitoring is safe and improves the functional outcome.

\section{AUTHOR CONTRIBUTIONS}

HF has performed literature research and put the topic in perspective of current knowledge. HF and W-DN have created the study design. AG and JZ gave support of creating the study design. Writing of the article has been performed by AG and JZ, as well of $\mathrm{HF}$ and $\mathrm{W}-\mathrm{DN}$.

\section{FUNDING}

The study received funding for the patient randomization by the German Society for Neurointensive Care and Emergency Medicine (DGNI).

subarachnoid hemorrhage and cerebral vasospasm. Stroke (2013) 44(8):215561. doi:10.1161/STROKEAHA.113.001015

12. Kim DH, Joseph M, Ziadi S, Nates J, Dannenbaum M, Malkoff M. Increases in cardiac output can reverse flow deficits from vasospasm independent of blood pressure: a study using xenon computed tomographic measurement of cerebral blood flow. Neurosurgery (2003) 53(5):1044-52. doi:10.1227/01. NEU.0000088567.59324.78

13. Mutoh T, Kazumata K, Terasaka S, Taki Y, Suzuki A, Ishikawa T. Early intensive versus minimally invasive approach to postoperative hemodynamic management after subarachnoid hemorrhage. Stroke (2014) 45(5):1280-4 doi:10.1161/STROKEAHA.114.004739

14. Ginsberg MD. Progress in neurobiology expanding the concept of neuroprotection for acute ischemic stroke: the pivotal roles of reperfusion and the collateral circulation. Prog Neurobiol (2016) 145-146:46-77. doi:10.1016/j. pneurobio.2016.09.002

15. Mazya MV, Ahmed N, Ford GA, Hobohm C, Mikulik R, Nunes AP, et al. Remote or extraischemic intracerebral hemorrhage - an uncommon complication of stroke thrombolysis: results from the safe implementation of treatments in stroke-international stroke thrombolysis register. Stroke (2014) 45(6):1657-64. doi:10.1161/STROKEAHA.114.004923

16. Álvarez-sabín J, Maisterra O, Santamarina E, Kase CS. Factors influencing haemorrhagic transformation in ischaemic stroke. Lancet Neurol (2013) 12(7):689-705. doi:10.1016/S1474-4422(13)70055-3

Conflict of Interest Statement: The authors declare that the research was conducted in the absence of any commercial or financial relationships that could be construed as a potential conflict of interest.

Copyright (c) 2017 Fuhrer, Günther, Zinke and Niesen. This is an open-access article distributed under the terms of the Creative Commons Attribution License (CC BY). The use, distribution or reproduction in other forums is permitted, provided the original author(s) or licensor are credited and that the original publication in this journal is cited, in accordance with accepted academic practice. No use, distribution or reproduction is permitted which does not comply with these terms. 